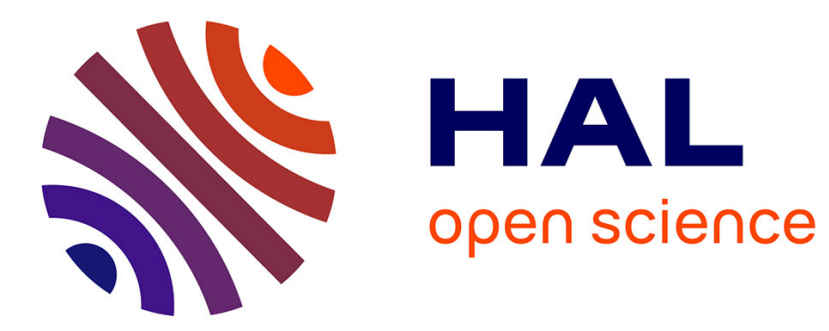

\title{
Cross-Layer based Congestion Control for WLANs
}

Lei Zhang, Patrick Sénac, Emmanuel Lochin, Michel Diaz

\section{To cite this version:}

Lei Zhang, Patrick Sénac, Emmanuel Lochin, Michel Diaz. Cross-Layer based Congestion Control for WLANs. 5th International ICST Conference on Heterogeneous Networking for Quality, Reliability, Security and Robustness, Jul 2008, Hong Kong, China. 10.4108/ICST.QSHINE2008.3845 . hal02864343

\section{HAL Id: hal-02864343 \\ https://hal.science/hal-02864343}

Submitted on 11 Jun 2020

HAL is a multi-disciplinary open access archive for the deposit and dissemination of scientific research documents, whether they are published or not. The documents may come from teaching and research institutions in France or abroad, or from public or private research centers.
L'archive ouverte pluridisciplinaire HAL, est destinée au dépôt et à la diffusion de documents scientifiques de niveau recherche, publiés ou non, émanant des établissements d'enseignement et de recherche français ou étrangers, des laboratoires publics ou privés. 


\section{Cross-Layer based Congestion Control for WLANs}

\author{
Lei Zhang \\ Université de Toulouse \\ ISAE - LAAS/CNRS \\ Toulouse, France \\ lei.zhang@isae.fr \\ Emmanuel Lochin \\ Université de Toulouse \\ ISAE - LAAS/CNRS \\ Toulouse, France \\ emmanuel.lochin@isae.fr
}

\author{
Patrick Sénac \\ Université de Toulouse \\ ISAE - LAAS/CNRS \\ Toulouse, France \\ patrick.senac@isae.fr \\ Michel Diaz \\ LAAS/CNRS \\ Université de Toulouse \\ Toulouse, France \\ diaz@laas.fr
}

\begin{abstract}
Congestion control is a fundamental mechanism for the stability of the Internet and is a central mechanism for TCP. However, this congestion control mechanism focuses mainly on the core network state and is blind to the characteristics of wireless and mobile access networks. Moreover, TCP window based congestion control ignores totally application layer QoS needs and entails throughput variations which are not compliant with application layer QoS constraints such as bandwidth, delay and jitter. The TCP-Friendly Rate Control protocol (TFRC) was originally designed in the context of wired networks. This protocol is one of the most convincing attempt to provide a congestion control mechanism adapted to multimedia flows, although limited in its capacity to fully address these issues. After an identification and evaluation of the subtle counterproductive interactions between the WLANs MAC layer and the transport layer, this paper shows a new approach towards congestion control for WLANs. This paper also introduces a specialization of TFRC (MTFRC: Mobile TFRC), which is adapted to wireless access networks. This TFRC specialization requires only slight changes to the standard TFRC protocol. Simulation results show substantial improvements for applications over TFRC in scenarios where the bottleneck situates on the MAC layer of the mobile nodes.
\end{abstract}

\section{Keywords}

TFRC, CSMA/CA, mobile network, MAC layer

\section{INTRODUCTION}

Recent researches $[3,5-7,10,12]$ have shown that TCP performs poorly over 802.11 because TCP was originally designed for wired network. In [2], the authors show when multiple TCP/UDP connections co-exist, the bandwidth of each TCP connection can be seriously degraded. The main reason is that the contention avoidance procedures implemented at the 802.11 MAC layer of access points slower the rate of returned ACK packets, which degrades the performance of ACK clocked protocols such as TCP. In [11], the authors lead an in-depth analysis on the TCP fairness over Wireless LAN, they conclude that the buffer size at the access point plays an important role in the observed unfairness. Even in a scenario that considers only TCP connections, the unfairness in TCP throughput ratio between upstream and downstream flows can be as large as 800 . Because of this TCP intrinsic potential of strong unfairness in the context of wireless access networks, TCP appears unadapted to WLANs.

Nowadays, multimedia continuous streams (e.g. video or audio streams) are dominantly accessed and exchanged by Internet users, TFRC protocol [8] has been proved to be able to offer a smooth, low delay and TCP-Friendly packet transmission in a wired network. Compared to TCP, it's a non feedback clocked protocol that potentially reduces unfairness issues raised by TCP under 802.11 [6]. To improve this mechanism over wireless networks, numerous research work aimed to find efficient differentiation algorithms (LDAs) to distinguish congestion errors from channel errors [1,4]. However, to date, few studies have focused on the influence of the contention based mechanism (CSMA/CA) to the TFRC protocol. In this paper we show that the rate processed by TFRC can strongly diverge from the rate offered by the Wifi MAC layer. This maladjustment between these two layers potentially induces dramatic loss rates.

In [9], Heusse and Al. analyze the performance anomaly of the IEEE 802.11 wireless local area networks, they conclude that mobile nodes with the lowest transmission rate impact on the performance of every mobile node in the coverage of the same access point. The resulting average throughput is principally restricted by the contention based MAC layer when the sending rate from transport layer becomes higher than the offered rate, packets can be lost in MAC buffers.

In this paper, we argues that coordinating the transport sending rate with the rate delivered by the WLAN MAC layer can entail an important loss reduction thus lowering end to end delays and jitter variations. We introduce a new specialization of TFRC: Mobile TFRC (MTFRC), which ef- 
ficiently adapts the sending rate from transport layer to the MAC layer. Furthermore, our method allows to solve the unfairness issues between upstream and downstream flows due to the MAC contention based mechanism. Our simulations show substantial improvements over TFRC in several wireless scenarios where congestion occurs at mobile nodes' MAC layer.

The rest of this paper is organized as follows: Firstly, in section 2, we give some simulation validated by experimental results to show the discrepancy between the transport layer bandwidth and the real sending rate delivered by the MAC level. Then in section 3, we analyze the performance of the $802.11 \mathrm{~b}$ DCF access method and give an analytical method to calculate the maximum throughput supported by the MAC layer for generic WLAN cases. We detail our proposed method MTFRC in section 4. We validate our analysis by means of simulation in section 5 , and section 6 concludes this study.

\section{EXPERIMENTAL DEMONSTRATION}

A set of simulations and experiments have been done to demonstrate the discrepancy between the transport layer sending rate and the real sending rate offered by the MAC layer.

The experimental testbed is composed of 3 hosts. Two of them are equipped with Atheros IEEE802.11a cards (to avoid interference by others $802.11 \mathrm{~b}$ hotspots situated in the lab). A mobile node A sends a UDP flow (packets size 1500 bytes) to a remote wired node B (receiver) via an access point (AP) which is $1 m$ distant from the mobile host. Both wireless stations run FreeBSD6.1 and use ifinfo tool in order to check the number of packets sent by the wireless interface. This tool returns information from the wireless card and in particular: the instantaneous length, the maximum length and the number of drops in the send queue of the wireless interface. The MAC buffer of node $\mathrm{A}$ is set to 50 packets. We measure the percentage of losses according to node A's sending rate. We made a similar simulation scenario with OPNET. The results of these simulations and experiments, as illustrated in Fig. 1, show that the throughput at the transport layer can reach or surpass the maximum bandwidth that the MAC layer can support and can lead to massive MAC buffer overflow and significant packet loss rate.

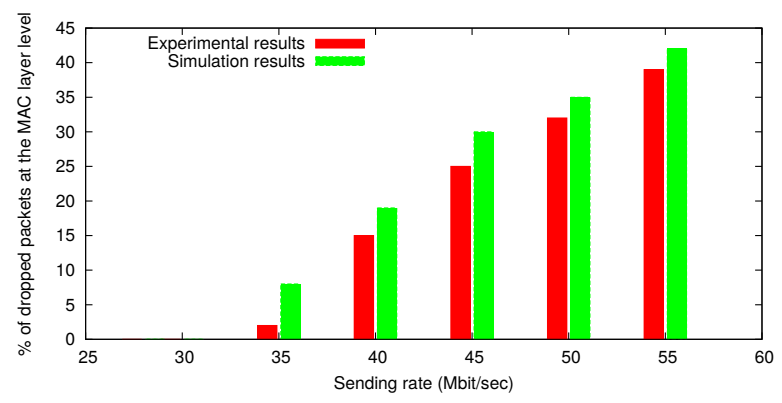

Figure 1: Percentage of packets lost on the MAC buffer as a function of the sending rate

\section{CALCULATION OF BANDWIDTH CAPAC- ITY LIMITED BY MAC LAYER}

In this section, we introduce an analytical model that makes possible for the transport entities above 802.11 MAC layer to accurately estimate the available throughput delivered by their WLAN MAC layer. The proposed model pushes further the approach proposed in [9] by considering on one side the diversity of mobile nodes' transmission rate profiles and on the other side, the specificities of TFRC flows.

\subsection{Data frame transmission duration}

We suppose $N$ mobile nodes (MN) in the same 802.11 b access point coverage that transmit data frames to remote hosts through the Access Point (AP). Four groups of mobile nodes can be distinguished among these $N$ mobile nodes. Indeed, according to their respective communication conditions, $N_{i}(i=1,2,3,4)$ mobile nodes use a transmission rate of $R_{i}$, where $R_{1}=11 \mathrm{Mb} / \mathrm{s}, R_{2}=5.5 \mathrm{Mb} / \mathrm{s}, R_{3}=2 \mathrm{Mb} / \mathrm{s}, R_{4}=$ $1 \mathrm{Mb} / \mathrm{s}$.

We denote $S$ : the MAC-layer frame length in bits. $T_{i}^{t r}$ represents the duration to transmit a data frame at a certain transmission rate $R_{i}$ :

$$
T_{i}^{t r}=\frac{S}{R_{i}} \quad R_{i} \in(1,2,5.5,11 \mathrm{Mb} / \mathrm{s})
$$

We denote $T_{i}^{o v}$ is a constant overhead which comprises DIFS, SIFS, two times of the PLCP preamble and the header transmission time as well as the MAC acknowledgment transmission time $t^{a c k}$.

$$
T_{i}^{o v}=D I F S+S I F S+2 * t_{i}^{p r}+t_{i}^{a c k}
$$

With $t^{p r}=192 \mu \mathrm{s}$ when the mobile node use the transmission rate of $1 \mathrm{Mb} / \mathrm{s}$ and $t^{p r}=96 \mu s$ when the mobile node uses the other transmission rates. In 802.11b, DIFS= $50 \mu s$, SIFS $=10 \mu s, t_{i}^{a c k}=\frac{112}{R_{i}}$.

We also denote $T^{c o n t}$, the average duration of backoff process. In [9], the authors give an approximation to express $T^{\text {cont }}$. (with $N$ : the total connected mobile nodes in the 802.11 coverage and $C W_{\min }$ : the minimum contention window size. In $802.11 \mathrm{~b}, C W_{\min }=31$ and $S L O T=20 \mu \mathrm{s}$.)

$$
T^{\text {cont }}(N)=\frac{S L O T *\left(1+P_{c}(N)\right) * C W_{\min }}{4 * N}
$$

Where $P_{c}(N)$ is the proportion of collisions experienced for each packet successfully acknowledged at the MAC level,

$$
P_{c}(N)=1-\left(1-\frac{1}{C W_{\min }}\right)^{(N-1)}
$$

Then, we have $T_{i}$ the overall duration for sending one data frame for each node in the group $i$ :

$$
T_{i}=T_{i}^{t r}+T_{i}^{o v}+T^{\text {cont }}(N)
$$

\subsection{Maximum throughput limited by contention based MAC layer for generic 802.11b sce- narios}


In this section, we focus on the calculation of the maximum bandwidth capacity delivered to mobile nodes by the MAC layer $\left(X_{m}\right)$ for generic $802.11 b$ scenarios. Firstly, we define greedy nodes, whose throughputs can reach or surpass the maximum bandwidth that the MAC layer can support. We have seen in section 2 that such a greedy behavior potentially causes congestion and losses on the MAC layer. Then, in contrast, we define rate sparing nodes, whose throughputs are limited by their application layer or by congestion over the network (i.g. a VoIP node that requires a relatively low bandwidth). In a contention based MAC layer, rate sparing nodes can alleviate the contention level in MAC layer and give more sending opportunities to other greedy nodes.

Following the definition in section 3.1, $N$ mobile nodes in $802.11 \mathrm{~b}$ coverage are classified into four groups $N_{i}(i=1,2,3,4)$ according to their transmission rates. For each group $N_{i}$, we also suppose that there are $K_{i}$ rate sparing nodes among $N_{i}$ mobile nodes using the transmission rate $R_{i}$. The average throughput of these $K_{i}$ nodes limited by their application or the congestion in the network is $X_{i}^{j}\left(j=1,2, \ldots, K_{i}\right)$.

Each of the $\left(N-\sum_{i=1}^{4} K_{i}\right)$ greedy nodes fully uses the maximum throughput $X_{m}$ delivered by the MAC layer. The aggregated bandwidth $X$ between all the mobile nodes and the access point is given by:

$$
X=\sum_{i=1}^{4} \sum_{j=1}^{K_{j}} X_{i}^{j}+\left(N-\sum_{i=1}^{4} K_{i}\right) * X_{m}+X_{A P}
$$

Note in this case, AP is considered as a normal contention based mobile node when it sends packets (i.e. TFRC feedback packets or downloaded data) to the $N$ mobile nodes.

We define $P_{i}^{j}=X_{i}^{j} / X\left(j=1,2, \ldots K_{i}\right)$, the proportion of throughput used by each rate sparing nodes in group $i$ and $P_{A P}=\frac{X_{A P}}{X}$, the proportion of the aggregated throughput used by the AP.

The proportion of the throughput for each of the $(N-$ $\left.\sum_{i=1}^{4} K_{i}\right)$ greedy mobile nodes is:

$$
P_{b}=X_{m} / X
$$

CSMA/CA protocol allows all the greedy mobile nodes to share fairly the radio channel. Theoretically, once a data frame is sent out by any of the greedy mobile nodes, it should wait a period of time to send another data frame, this average time $T$ between the two successive emission packets comprises the following 4 parts: (1) the time required for sending one packet by each of the greedy node with different transmission rate: $\sum_{i=1}^{4} T_{i} *\left(N_{i}-K_{i}\right) ;(2)$ the time required for sending packets by the sparing nodes with different transmission rate. According to the above analysis on the rate proportion, every time a packet is sent out by a greedy node, there should be $\left(\sum_{i=1}^{4} \sum_{n=1}^{K_{i}} P_{i}^{n}\right) / P_{b}$ packets sent by all the rate sparing nodes; (3) the time required for sending packets (i.e. TFRC feedback packets or downloading data) from the AP to $N$ mobile nodes. Similarly, every time a packet is sent out by a greedy node, there should be $\left(P_{A P} / P_{b}\right)$ packets sent by AP; $(4)$ the time spent in collisions $\left(T_{c o l}\right)$.

$$
\begin{aligned}
& T=\sum_{i=1}^{4} T_{i} *\left(N_{i}-K_{i}\right)+\frac{\sum_{i=1}^{4} \sum_{n=1}^{K_{i}} P_{i}^{n} * T_{i}}{P_{b}}+ \\
& T_{\text {col }}+T_{A P} * \frac{P_{A P}}{P_{b}}
\end{aligned}
$$

In the generic scenario, since AP is considered as a normal transmission mobile node, $T_{A P}$ and $T_{i}$ can be calculated with equation (5), and we have a total of $(N+1)$ contention mobile node.

$$
T_{c o l}=P_{c}(N+1) * t_{j a m} *(N+1)
$$

$t_{j a m}$ represents the average time spent in collision for each node in case of collision, whose calculation is given in appendix 7.3 .

Since the average time between the two successive emission packets is $T$, we can calculate $X_{m}$, the maximum throughput supported by the MAC layer for greedy nodes with the following equation:

$$
X_{m}=S / T
$$

With $S$ the length of MAC layer packet in bits.

The maximum available throughput at the transport layer is:

$$
X_{t}=S_{t} / T
$$

With $S_{t}$ the length of transport layer packet in bits.

\subsection{TFRC and UDP cases with greedy nodes only}

Based on the above calculation for the generic scenarios, this section discusses two special cases: TFRC and UDP cases with greedy nodes only. Since there is no rate sparing nodes in these two cases, we have $K_{i}=0, P_{i}^{n}=0$.

\subsubsection{In TFRC case}

Based on the equation (8), $T$ becomes:

$$
T=\sum_{i=1}^{4}\left(T_{i} * N_{i}\right)+T_{c o l}^{\prime}+T_{A P} * \frac{P_{A P}}{P_{b}}
$$

Where $T_{c o l}^{\prime}$ is the time spent in collisions given by: 


$$
T_{\text {col }}^{\prime}=P_{c}(N+1) * t_{j a m}^{\prime} *(N+1)
$$

The calculation of $t_{j a m}^{\prime}$, the average time spent in collision for each node in TFRC case is given in appendix 7.2.

According to the above proportion analysis, we have:

$$
\frac{P_{A P}}{P_{b}}=\frac{X_{A P}}{X_{m}}
$$

Then the maximum bandwidth capacity supported by mobile nodes' MAC layer $X_{m}$ in TFRC mode can be calculated with equations (10), (12), (13), (14).

If we suppose there are only TFRC upload flows in our scenario and the TFRC receiver returns a feedback packet every $R T T$, we can calculate the bandwidth $X_{A P}$ (corresponding to the TFRC feedback packets) from the AP to the $N$ mobile nodes as follows:

$$
X_{A P}=\frac{N}{R T T} * S_{f}
$$

Where, $S_{f}$ is the size of TFRC feedback packet (816 bits) and $R T T$ the averaged round trip time.

\subsubsection{In UDP case}

If we suppose only UDP upload flows exist in our scenario, we have $P_{A P}=0$, then $T$ can be simplified to:

$$
T=\sum_{i=1}^{4}\left(T_{i} * N_{i}\right)+T_{c o l}^{\prime \prime}
$$

Note in this case, since AP is no longer considered as a transmission mobile node (no download packets are sent from AP to $\mathrm{N}$ mobile nodes), we have:

$$
T_{\text {col }}^{\prime \prime}=P_{c}(N) * t_{j a m}^{\prime \prime} * N
$$

Where the calculation $T_{j a m}^{\prime \prime}$ is given in appendix 7.1.

Thus, the maximum bandwidth capacity supported by mobile nodes' MAC layer $X_{m}$ (and $X_{t}$,maximum available bandwidth at the transport layer) in UDP mode can be calculated with equations (10), (11), (16), (17).

Our analytical model has been validated by a set of simulations under OPNET. Figure 2 shows the evolution of $X_{t}$ (with analytical and simulation results) in UDP case in terms of number of the uploading mobile nodes $(N=[4,30])$ in four different scenarios. In the first scenario, all the mobile nodes have a transmission rate of $11 \mathrm{Mbit} / \mathrm{s}$. Then, for each other three scenarios, one among $N$ mobile nodes has

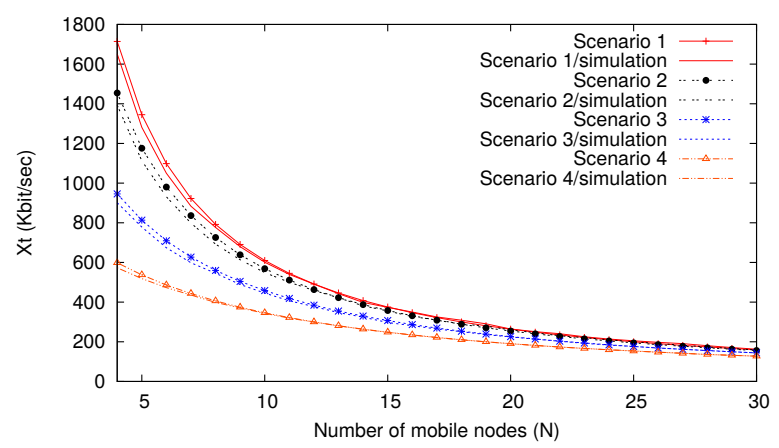

Figure 2: Evolution of $X_{t}$ in UDP mode as a function of the number of uploading mobile nodes

respectively a transmission rate of $5.5 \mathrm{Mbit} / \mathrm{s}, 2 \mathrm{Mbit} / \mathrm{s}$ and $1 \mathrm{Mbit} / \mathrm{s}$.

Otherwise, TFRC feedback packets take a small part of bandwidth resource from the aggregated bandwidth capacity $(X)$. If we suppose that $X$ remains the same for UDP and TFRC scenarios where only upload flows exist (with the same distribution of $N_{i}$ ). We can approximately estimate the maximum available throughput at the transport layer:

$$
X_{t}^{U D P}=X / N
$$

for UDP case and:

$$
X_{t}^{T F R C}=\left(X-X_{A P}\right) / N
$$

for TFRC case. The relative error between the two available bandwidth is :

$$
\epsilon=\frac{X_{t}^{U D P}-X_{t}^{T F R C}}{X_{t}^{U D P}}=\frac{X_{A P}}{X}=\frac{S_{f} * N}{R T T * X}
$$

This relative error $\epsilon$ is normally much less than $5 \%$ in the context of $802.11 \mathrm{~b}$. We can integrate the UDP based model into the TFRC scenarios to simplify the calculation when the relative error $\epsilon$ is little.

\section{CROSS-LAYERED CONGESTION CON- TROL}

\subsection{Rate adaptation}

In section 3, we have given formulas to find the maximum throughput supported by contention based MAC layer for the mobile nodes. When the sending rate from transport layer (eg. estimated by TFRC equation) becomes higher than the bandwidth offered by the MAC layer, packets can be lost in the MAC buffers. These losses increase the loss event rate $p$ processed by the TFRC protocol and degrade the TFRC sending rate. However, following a phase without losses, the TFRC sending rate will increase until it exceeds the available MAC layer rate again, thus inducing harmful variations and unstable oscillations of the sending rate. In order to illustrate this behavior, we simulate two mobile nodes uploading data to remote servers with a transmitting rate of $5.5 \mathrm{Mb} / \mathrm{s}$ where congestion occur at the MAC 
layer. Fig 3 gives the result of the TFRC throughput and the maximum available throughput at the transport layer $\left(X_{t}\right)$. We can observe that TFRC obtains unstable rate variations around $X_{t}$ and that MAC buffer overflow occurs. The transport layer throughput has a standard deviation of $115.8 \mathrm{~Kb} / \mathrm{s}$ for an average throughput of $1.94 \mathrm{Mb} / \mathrm{s}$ after $t=25 \mathrm{sec}$

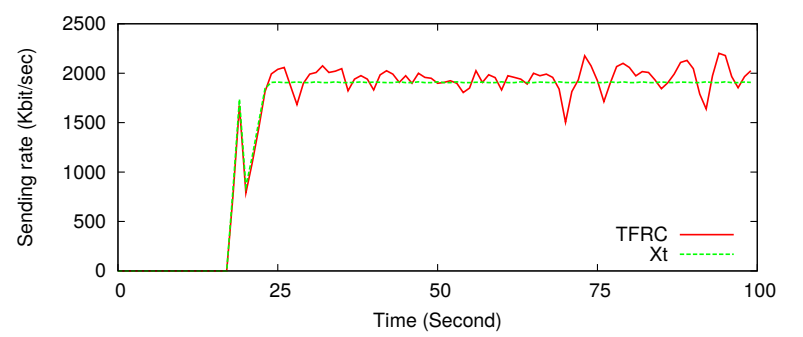

Figure 3: Performance comparison between TFRC and $X_{t}$

In order to improve the QoS delivered to TFRC flows on WLAN access networks, we introduce a specialization of the TFRC protocol (MTFRC) to WLANs based on a cross-layer interaction between the transport and the mac layers. More precisely, we propose to constraint the TFRC rate equation with the MAC layer available rate processed as defined in the previous section. The algorithm for processing the MAC limited threshold $X_{t}$ is inserted in every access point AP, this threshold can be calculated according to different dynamical parameters which are collected by analyzing the PLCP frame fields of received packets by AP in real time. Sending and receiving rate profiles are also taken into account in the case when rate sparing nodes exist. Every mobile node then compares its processed TFRC equation based sending rate $X_{t f r c}$ with the threshold $X_{t}$. If the calculated $X_{t f r c}$ is higher than $X_{t}$, the sending rate $X_{\text {send }}$ is then adjusted to $X_{t}$ to avoid congestion and losses in the MAC layer.

$$
X_{\text {send }}=\min \left(X_{t f r c}, X_{t}\right)
$$

\subsection{Fairness improvement}

Since the access point, AP, is considered as a normal contentionbased mobile node, it has the same opportunity of sending packets (to all the download mobile nodes) as any of the other upload mobile nodes. Indeed, if we suppose that $U$ TFRC mobile nodes are uploading streams to remote servers via $A P$, and that $D$ TFRC flows are concurrently sent by this same access point to $D$ TFRC downloading mobile nodes, then the average throughput of each upload flow is equal to the aggregate throughput of the download flows sent by AP. In other words, instead of getting a fair mean throughput ratio of $D /(U+D)$, the aggregated download flow receive a unfair throughput ratio of $1 /(U+1)$.

We introduce in this section a rate-equalization mechanism uniquely based on an end to end mechanism (i.e. with no modification of the MAC layer) that makes it possible for the downloading flows to gain a fair share of the throughput delivered by the MAC layer.

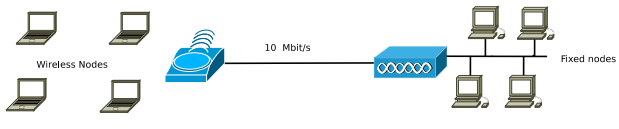

Figure 4: Simulation topology

Following the analysis in section 3, since AP is considered as a normal transmission mobile node, the average uploading bandwidth supported at MAC layer $X_{u}$ can be estimated from equation (10) (where $N=U+1$ ). Note that $X_{u}$ is equal to $X_{m}=D * X_{d}$ where $X_{d}$ is the average bandwidth for each downloading node. The aggregated bandwidth $(X)$ exchanging between the AP and all the mobile nodes is given by:

$$
X=U * X_{u}+D * X_{d}=(U+1) * X_{m}
$$

The object of the proposed rate equalization mechanism is to assign this total bandwidth $X$ more fairly to each of the mobile node (download or upload mobile nodes). So each mobile node can get a bandwidth of

$$
X_{\text {fair }}=\frac{X}{(U+D)}=\frac{X_{m} *(U+1)}{(U+D)}
$$

Therefore, by combining both the fair share constraint and the MAC rate constraint in equation (19), we obtain the constrained sending rate:

$$
X_{\text {send }}=\min \left(X_{t f r c}, X_{t}, X_{\text {fair }}\right)
$$

This specialization of TFRC limits the sending rate of each of the $U$ upload mobile node to $X_{\text {fair }}$. Therefore, the contention based mechanism allows AP to gain more sending opportunities, which corresponds to an additional bandwidth of $\left(X-U * X_{\text {fair }}\right)$ for the AP. Thus, each download node can get a bandwidth of $X_{\text {fair }}$. Simulation results, as shown in section 5 , demonstrate that such an end to end approach ensures a fair share of the bandwidth between upload and download flows.

\section{SIMULATION AND VALIDATION}

We have simulated under OPNET a set of wireless scenarios to validate our proposed method. We present in this section three typical scenarios. In all the scenarios, we set the link bandwidth capacity of the access router to $C=10 \mathrm{Mb} / \mathrm{s}$ in order to have $C>>\sum_{1}^{N} X_{m}$ with $N$ the number of mobile nodes. As a result, $X_{m}$ is considered as the bottleneck between the mobile node and the destination.

We suppose that several TFRC mobile nodes send data packets to their corresponding servers via an $802.11 \mathrm{~b}$ access router (see Fig. 4). The buffer size in the access point is 20 KByte and the buffer size of MAC layer of each mobile node is 256 Kbit (default setting in OPNET). The 
size of data frame $\left(S_{t}\right)$ is equal to 8192bit (MPDU size $S=8614 b i t)$. Since the bandwidth corresponding to TFRC feedback packets is negligible, $X_{t}$ is estimated in UDP mode in order to simplify the calculation (see section 3.3.2. In all the simulations, the traffic generation starts at $t=15 \mathrm{sec}$.

\subsection{Scenario1}

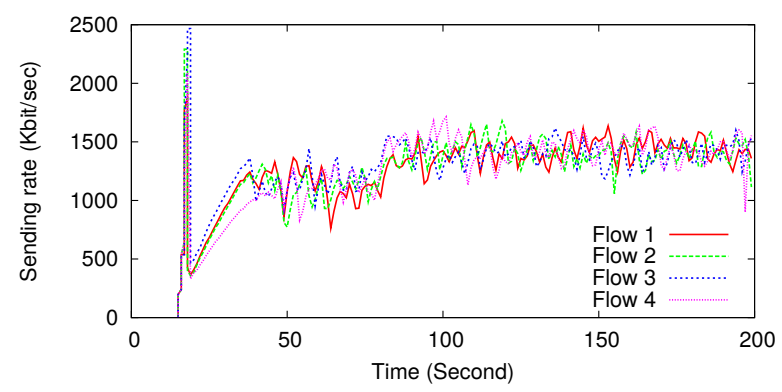

Figure 5: TFRC sending rate

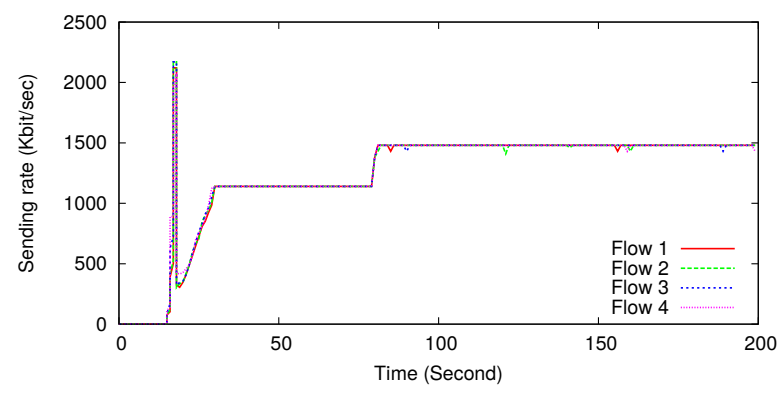

Figure 6: MTFRC sending rate

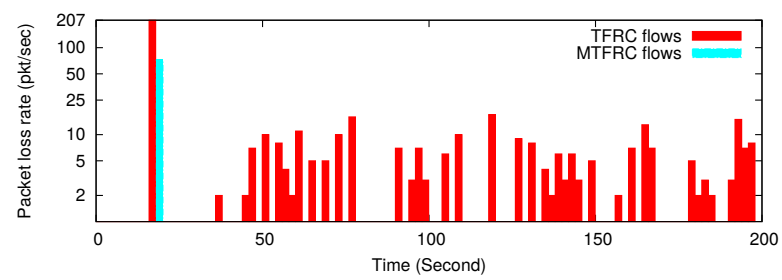

Figure 7: Packet loss rate of TFRC and MTFRC aggregates

In scenario 1, we suppose that two of the mobile nodes always share a transmission rate of $11 \mathrm{Mb} / \mathrm{s}$. The other two mobile nodes have a transmission rate of $5.5 \mathrm{Mb} / \mathrm{s}$ between $t=[15 \mathrm{sec}, 80 \mathrm{sec}]$. When they move towards the access router, their transmission rates turn to $11 \mathrm{Mb} / \mathrm{s}$ at $t=80 \mathrm{sec}$. Between $t=[15 \mathrm{sec}, 80 \mathrm{sec}]$, according to equation (11) (with $S_{t}=1024$ Byte, $\left.N_{1}=2, N_{2}=2, N=4\right), X_{t}$ is equal to $1.14 \mathrm{Mb} / \mathrm{s}$ between $t=[80 \mathrm{sec}, 200 \mathrm{sec}]$ and $X_{t}$ rises to $1.48 \mathrm{Mb} / \mathrm{s}$ with $N_{1}=4$. In this scenario, the sending rate $X_{\text {send }}$ always equals to $X_{t}$ because the bottleneck always situates on the MAC layer of each mobile node. Fig. 5 and 6 represents the sending rate of TFRC and MTFRC. Fig. 7 returns the sum of the packet loss rate of each TFRC and MTFRC flows. After the slowstart phase, on average, 1.51 packets per second are dropped by the MAC layer for the TFRC flows while zero packets are dropped for MTFRC flows as shown Fig. 7. We can see that our proposal efficiently avoids the losses on the MAC layer and substantially improves the quality of the transmission.

\subsection{Scenario 2}

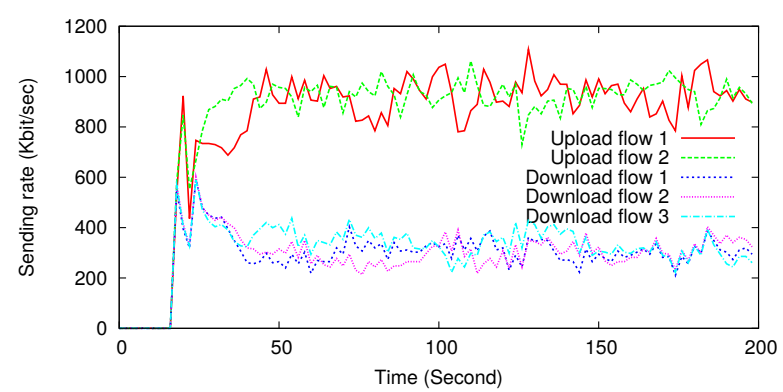

Figure 8: TFRC sending rate

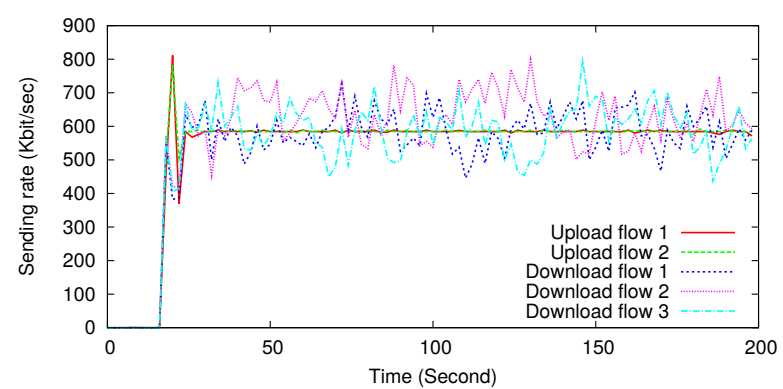

Figure 9: Sending rate with MTFRC

In scenario 2, we address fairness issues between upload and download flows. For illustration purpose, we consider that two mobile nodes upload TFRC flows (of which the transmission rates are respectively $11 \mathrm{Mb} / \mathrm{s}$ and $2 \mathrm{Mb} / \mathrm{s}$ ) and three mobile nodes download TFRC flows with transmission rate of $5.5 \mathrm{Mb} / \mathrm{s}$. Fig. 8 shows that by default, each upload flow occupies much more bandwidth (average ratio of three) than each download flow. Conversely, when using MTFRC improved with the proposed fairness mechanism, we observe a fair share of the bandwidth between the upload and download flows (Fig. 9). Indeed, in this case, when applying equation (11) (21) and (22), since the AP is considered as a upload node we have $N=3, N_{1}=N_{3}=1, N_{2}=1$ (which corresponds to the aggregated 3 download nodes) and we get $X_{u p}=X_{m}=968 \mathrm{~Kb} / \mathrm{s}$ and $X_{\text {fair }}=X_{u p} * 3 / 5=581 \mathrm{~Kb} / \mathrm{s}$. The sending rate for each of the upload mobile nodes is then limited to $X_{\text {fair }}$ to allow download nodes sharing the same bandwidth.

\section{CONCLUSION}

In this paper, we have presented experimental tests showing the lack of adaptation mechanism between the transport and the WLAN MAC layers. This discrepancy can lead to MAC buffer overflow. In order to solve this problem, the present proposal makes the transport layer aware of the underlying MAC layer supported rate. This cross-layered approach is based on an analytical estimation of the instantaneous available throughput supported by MAC layer. Then, the result 
is given as a parameter to the transport layer (e.g. TFRC rate calculation) in order to suppress losses in MAC buffers, therefore improves significantly the transmission quality and can also decrease the number of retransmission if reliability mechanisms are involved. The resulting WLAN specialization of TFRC, MTFRC, entails a dynamic adjustment of the TFRC sending rate according to the number and transmission state of the whole set of mobile nodes attached to the same WLAN hotspot. Moreover, we pushed forward the idea of rate adjustment according to the WLAN communication status by introducing a new end to end approach for improving the fairness between uploading and downloading flows. One of the advantages of this proposal is that it requires only slight changes to the standard TFRC protocol and no change at the MAC layer. Our simulation results show substantial improvements over TFRC in several representative wireless scenarios. Our future work aims to determine a light cost and more efficient information collection method involved in the access point. Furthermore, we will investigate the potential impacts of the proposed approach in the context of handover managements.

\section{APPENDIX}

\subsection{Estimate of $t_{j a m}^{\prime \prime}$ in UDP mode}

In this section, we focus on the calculation of the average time spent in collision $\left(t_{j a m}^{\prime \prime}\right)$ to send one frame for each mobile node.

When a mobile node (MN) of the $N_{i}$ nodes wants to send out one frame, it risks being deferred because it exists a probability of collisions $P_{c}(N)$ (proportion of collisions, see section 3). If the collision happens, the node which causes MN deferring can be one of the other $\left(N_{i}-1\right)$ mobile nodes in group $N_{i}$ with a probability of $\left(N_{i}-1\right) /(N-1)$, in this case, $\mathrm{MN}$ has to wait minimum $T_{i}$ to prepare resending; the node can also be one of the $N_{j}$ nodes in group $j(j \neq i)$ with a probability of $N_{j} /(N-1)$, in this case, MN has to wait minimum $T_{j}$ to prepare resending. So for any of the node in group $N_{i}$, the average time spent in collision to send one frame is expressed by the following equation:

$$
t_{j a m}^{i}=\frac{N_{i}-1}{N-1} * T_{i}+\sum_{j=1}^{4, j \neq i} \frac{N_{j}}{N-1} * T_{j}
$$

Here, we try to find the average time spent in collision for each of the $N$ nodes, so:

$$
t_{j a m}^{\prime \prime}=\frac{N_{1}}{N} * t_{j a m}^{1}+\frac{N_{2}}{N} * t_{j a m}^{2}+\frac{N_{3}}{N} * t_{j a m}^{3}+\frac{N_{4}}{N} * t_{j a m}^{4}
$$

With the above equations, we have:

$$
t_{j a m}^{\prime \prime}=\frac{\sum_{i=1}^{4}\left(N_{i} * T_{i}\right)}{N}
$$

7.2 Estimate of $t_{\text {jam }}^{\prime}$ in the TFRC greedy mode Similar to the calculation in section 7.1, for a mobile node $(\mathrm{MN})$ in group $N_{i}$, if a collision happens, the node which causes MN deferring can be one of the other $\left(N_{i}-1\right)$ mobile nodes in group $N_{i}$ with a probability of $\left(N_{i}-1\right) * X_{m} /((N-$ $\left.1) * X_{m}+X_{A P}\right)$, the node can also be one of the $N_{j}$ nodes in group $j(j \neq i)$ with a probability of $N_{j} * X_{m} /((N-1) *$ $\left.X_{m}+X_{A P}\right)$, the node can also be the AP with a probability of $X_{A P} /\left((N-1) * X_{m}+X_{A P}\right)$. So similarly, the average time spent in collision for AP and every node in group $N_{i}$ to send one frame is:

$$
\begin{aligned}
t_{j a m}^{i} & =\frac{\left(N_{i}-1\right) * X_{m}}{(N-1) * X_{m}+X_{A P}} * T_{i}+ \\
& \sum_{j=1}^{4, j \neq i} \frac{N_{j} * X_{m}}{(N-1) * X_{m}+X_{A P}} * T_{j}+ \\
& \frac{X_{A P} * T_{A P}}{(N-1) * X_{m}+X_{A P}}
\end{aligned}
$$

For the AP:

$$
t_{j a m}^{A P}=\sum_{j=1}^{4} \frac{N_{j} * X_{m}}{(N-1) * X_{m}+X_{A P}} * T_{j}
$$

The average time spent in collision for each of the $N+1$ mobile nodes (including AP) is:

$$
t_{j a m}^{\prime}=\sum_{j=1}^{4} \frac{N_{i}}{N+1} * T_{j a m}^{i}+\frac{1}{N+1} * T_{j a m}^{A P}
$$

Finally, we have approximately:

$$
t_{j a m}^{\prime}=\frac{\sum_{i=1}^{4}\left(N_{i} * X_{m} * T_{i}\right)+X_{A P} * T_{A P}}{N * X_{m}+X_{A P}}
$$

\subsection{Estimate of $t_{j a m}$ in the rate sparing mode for the non-frequent feedback based pro- tocols}

We denote $P_{i}^{m}$ the proportion of throughput for each mobile node in group $N_{i}$ ( $m$ is the index of the mobile nodes in each group $N_{i}$ ). So $P_{i}^{m}$ represents the proportion of throughput for both the rate sparing nodes and the greedy nodes. For each mobile node, if its proportion of throughput increases, this node has upper hand for the contention on the MAC layer. For example, if this proportion is $\mathrm{q} \%$, the collisions caused by this node has a probability of $\mathrm{q} \%$.

Similar to the analysis in appendix 7.1, for each node in group $N_{i}$, the collision can be caused by the other $\left(N_{i}-1\right)$ nodes or the nodes in other groups $N_{j}(j \neq i)$. The average time spent in collision to send out one frame for AP and any of the node in group $N_{i}$ can be expressed by the following equation: 


$$
\begin{gathered}
t_{j a m_{i}}^{m}=\frac{\sum_{k=1, k \neq m}^{N_{i}}\left(P_{i}^{k} * T_{i}\right)+\sum_{j=1}^{4, j \neq i} \sum_{k=1}^{N_{j}}\left(P_{j}^{k} * T_{j}\right)}{\left(1-P_{i}^{m}\right)} \\
t_{j a m}^{A P}=\frac{\sum_{j=1}^{4} \sum_{k=1}^{N_{j}}\left(P_{j}^{k} * T_{j}\right)}{1-P_{A P}}
\end{gathered}
$$

Finally, the average time spent in collision for each of the $N$ nodes is:

$$
t_{j a m}=\sum_{i=1}^{4}\left(\sum_{m=1}^{N_{i}} P_{i}^{m} * t_{\text {jam }}^{m}\right)+P_{A P} * t_{j a m}^{A P}
$$

\section{REFERENCES}

[1] V. Arya and T. Turletti. Accurate and Explicit Differentiation of Wireless and Congestion Losses. In MWN Workshop on Mobile and Wireless Networks, 2003.

[2] R. Bruno, M. Conti, and E. Gregori. Design of an enhanced access point to optimize TCP performance in Wi-Fi hotspot networks. Wireless Networks, 13(2), Apr. 2007.

[3] F. Cali, M. Conti, and E. Gregori. Dynamic Tuning of the IEEE 802.11 Protocol to Achieve a Theoretical Throughput Limit. IEEE/ACM Transactions on Networks, 8(6), 2000.

[4] S. Cen, P. C. Cosman, and G. M. Voelker. End-to-End Differentiation of Congestion and Wireless Losses. IEEE/ACM Transactions on Networks, 11(5), 2003.

[5] K. Chen, Y. Xue, and K. Nahrstedt. On Setting TCP's Congestion Window Limit in Mobile Ad Hoc Networks. In Proc. IEEE Intl. Conf. on Communications (ICC), 2003.

[6] Z. Fu, P. Zerfos, H. Luo, S. Lu, L. Zhang, and M. Gerla. The Impact of Multihop Wireless Channel on TCP Throughput and Loss. In Proc. of IEEE Infocom, 2003.

[7] M. Gerla, K. Tang, and R. Bagrodia. TCP performance in wireless multi-hop networks. In Proc. of IEEE WMCSA, 1999.

[8] M. Handley, S. Floyd, J. Pahdye, and J. Widmer. TCP-Friendly Rate Control (TFRC): Protocol Specification. Request For Comments 3448, IETF, Jan. 2003.

[9] M. Heusse, F. Rousseau, G. Berger-Sabbatel, and A. Duda. Performance Anomaly of 802.11b. In Proc. of IEEE Infocom, 2003.

[10] G. Holland and N. H. Vaidya. Analysis of TCP Performance over Mobile Ad Hoc Networks. In in Proc. of IEEE/ACM MOBICOM, 1999.

[11] S. Pilosof, R. Ramjee, D. Raz, Y. Shavitt, and P. Sinha. Understanding TCP fairness over Wireless LAN. 2003 IEEE. In Proc. of IEEE Infocom, 2003.

[12] V. Ramarathinam and M. A. Labrador. Performance Analysis of TCP over Static Ad Hoc Wireless Networks. In Proc. of the ISCA PDCS, 2000. 